\title{
Circuit
}

Musiques contemporaines

\section{Introduction : John Rea, la raison des forces mouvantes}

\section{Jonathan Goldman}

Volume 26, numéro 1, 2016

John Rea : une masquographie raisonnée

URI : https://id.erudit.org/iderudit/1036055ar

DOI : https://doi.org/10.7202/1036055ar

Aller au sommaire du numéro

Éditeur(s)

Les Presses de l’Université de Montréal

ISSN

1183-1693 (imprimé)

1488-9692 (numérique)

Découvrir la revue

Citer ce document

Goldman, J. (2016). Introduction : John Rea, la raison des forces mouvantes. Circuit, 26(1), 5-8. https://doi.org/10.7202/1036055ar d'utilisation que vous pouvez consulter en ligne.

https://apropos.erudit.org/fr/usagers/politique-dutilisation/ 


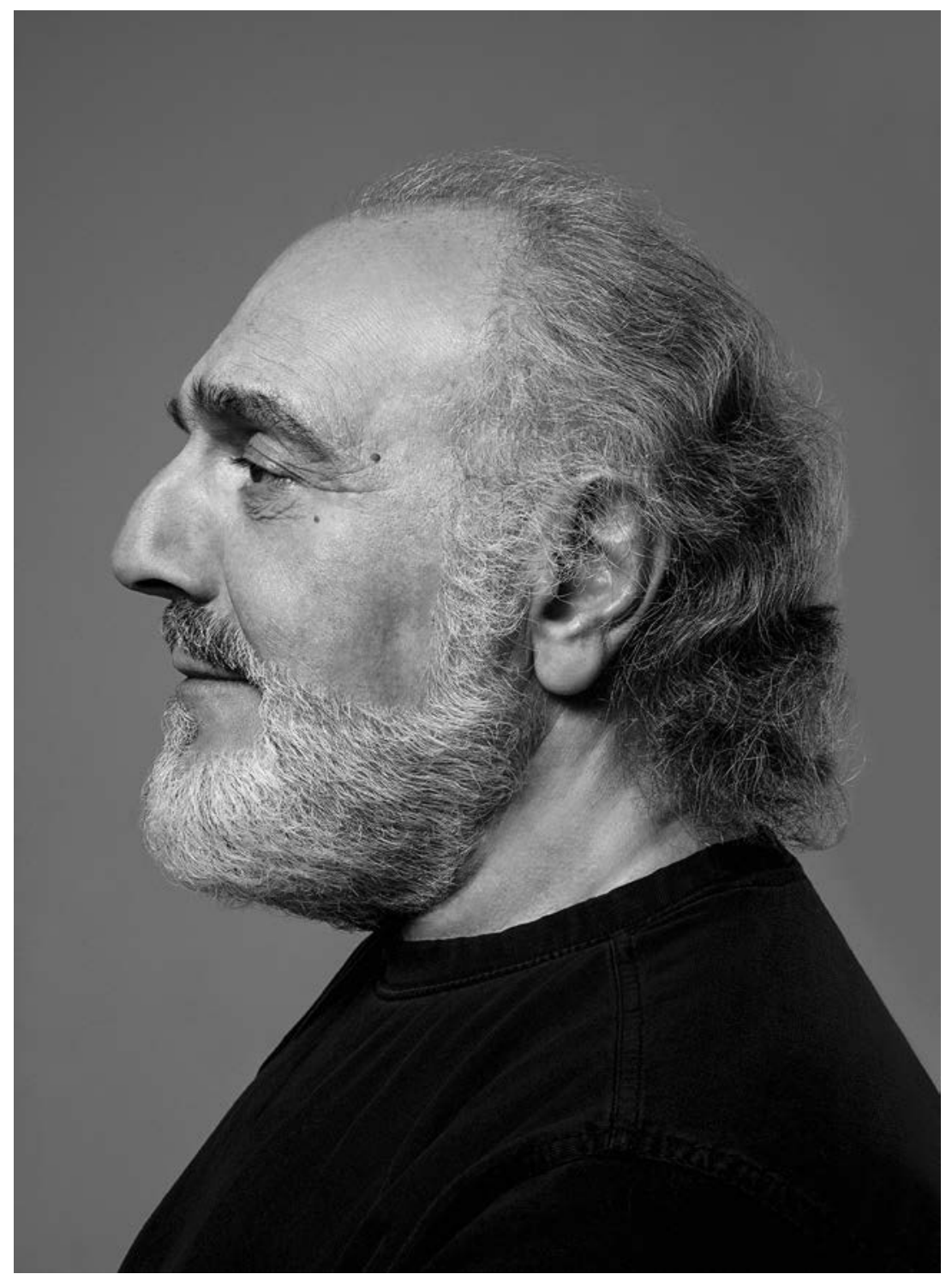

John Rea, 2015, par Justine Latour pour la Série hommage sMcQ. 


\section{Introduction : John Rea, la raison des forces mouvantes}

Jonathan Goldman

Consacrer un numéro à John Rea n'est pas chose aisée: si les collaborateurs de Circuit ont l'habitude de traduire en mots les sons inouïs d'œuvres de création, le musicien qu'est Rea est tout sauf silencieux quant aux couches de significations que recouvrent ses œuvres. Non seulement le compositeur montréalais s'empare souvent de sa plume pour théoriser subtilement sa musique dans des écrits d'ampleur, mais il s'avère aussi que ses œuvres sont invariablement le fruit d'une réflexion aboutie ou d'une autoréflexivité assumée - pour ne pas dire, parfois, l'acte même de réfléchir incarné en sons! La réflexion de Rea en musique porte invariablement sur les significations que peut abriter une œuvre, qu'elle soit musicale (Wagner, Mahler, Schoenberg, Berg, Ligeti, Grisey), littéraire (Dante, Poe, Baudelaire, Goethe, Pessoa) ou picturale (Kokoschka, Vasarely, Escher).

Il est doublement difficile pour l'équipe de Circuit d'aspirer à une attitude objective lorsqu'il est question de John Rea. Membre fondateur du Comité de rédaction de la revue, il a alimenté nombre de discussions de 1990 à 2011, en plus d'avoir signé 17 articles. John Rea fait partie de la famille de Circuit depuis la première heure et il a laissé une trace indélébile sur les numéros à travers plus de deux décennies. En plus d'avoir publié ses textes ainsi qu’un numéro «Carte blanche à Bouliane et Rea » (vol. 9, nº 2, 1998), la revue s'est penchée sur la musique du compositeur, quoique à une moindre mesure, une importante exception étant la deuxième édition du (désormais incontournable !) Cahier d'analyse, dans le vol. 17, $\mathrm{n}^{\circ} 2$ (2007), où James Galaty propose un parcours analytique de l'œuvre pour orchestre Hommage à Vasarely (1977). C'est donc dans le prolongement de cet article que Circuit propose un numéro entier consacré à l'œuvre de John Rea, et tente de prendre la mesure de l'œuvre et de la pensée du compositeur en invitant le lecteur à suivre le fil de ce créateur multiforme. 
Ce nouvel opus de la revue paraît, rappelons-le, dans un contexte bien précis. Tous les deux ans depuis 2008, et en alternance avec le festival Montréal/Nouvelles Musiques (MNM), la Société de musique contemporaine du Québec (SMCQ) consacre sa saison artistique à l'œuvre d'un seul compositeur. Dans le cadre de cette Série hommage, la SMCQ et son directeur artistique, Walter Boudreau, invitent des dizaines d'organismes de musique contemporaine à sunir à elle pour célébrer la production de ce créateur. On se souvient encore de l'année Claude Vivier, premier compositeur «hommagé » en 2007-2008, suivi, deux ans plus tard, par son maître Gilles Tremblay en 2009-2010, par la compositrice Ana Sokolović en 2011-2012, et par Denis Gougeon il y a de ça deux ans. Nous voilà donc en 2015-2016 et les projecteurs se dirigent vers l'œuvre abondante et fort diversifiée de John Rea. En plus de diffuser des œuvres existantes, la Série hommage met en place un dispositif de commande du compositeur célébré. C’est ainsi qu’au cours de la saison, l'Orchestre symphonique de Montréal (OSM) a commandé le triptyque pour orgue Épris de liberté, Souffles de liberté et Héleurs de liberté (2015), qui sera créé par l'organiste attitré de l'orchestre, Jean-Willy Kunz. Pour sa part, le Nouvel Ensemble Moderne (NEM, Lorraine Vaillancourt, dir.) interprètera La chute des anges rebelles en avril 2016, tandis que deux œuvres ont été spécialement conçues par Rea pour des ensembles écoliers: Pincer, penser, chanter et Danser avec la gravité.

Comment trouver un fil conducteur à travers ce jardin musical luxuriant aux multiples formes? Il nous semble qu'une des portes d'entrée à l'œuvre du musicien réside dans une réflexion sur le concept de la médiation. Chez John Rea, le médium, le matériau, n'est jamais pris pour acquis, et il n'est pas anodin de rappeler qu'un séminaire qu'il a récemment proposé à l'École de musique Schulich de l'Université McGill (Montréal) - son attache institutionnelle depuis le début des années 1970 - porte justement sur le matériau. Dans l'œuvre Hommage à Vasarely, par exemple, qui vient d'être évoquée, Rea est d'abord saisi par la façon dont le peintre hongrois projette des figures géométriques sur le plan du tableau tout en les déformant: les projections ainsi altérées constituent donc un premier niveau de médiation. Par la suite, le compositeur ajoute une seconde couche sémantique pour faire de l'œuvre un hommage subreptice à Iannis Xenakis, et ce, en pensant aux projections proposées par celui-ci de l'espace architectural sur l'espace temporel du son dans une œuvre emblématique comme Metastaseis (1953-1954), ou bien dans ses Polytopes, alliant musique, architecture et lumière. Rea conçoit pour son hommage une projection unique de l'espace d'un tableau sur l'espace de l'orchestre (sur fond d'un «canevas blanc» constitué d'un accord constitué 
de 11 quintes superposées). Dans la troisième partie d'une œuvre plus récente, Accident: tombeau de Grisey (2004), Rea médite sur les projections que proposait le courant spectral de l'acoustique sur le musical, en prenant comme point de départ l'œuvre emblématique du compositeur français disparu six ans auparavant, Partiels (1975). Dans l'œuvre magistrale au titre opulent J'ignore si j'étais un homme rêvant alors que j'étais un papillon ou si je suis à présent un papillon rêvant que je suis un homme (ou simplement Homme papillon) (2001), le musicien explore la zone grise qui sépare l'artisanat de l'algorithmel. Enfin, une ouvre parmi tout le catalogue du compositeur renvoie à la médiation à même son titre: Médiator (...pincer la musique aujourd'hui...) (1981), composée pour divers instruments à cordes pincées et entièrement notée en tablatures. Le terme choisi fait ainsi référence à son homonyme latin pour désigner le plectre, cet objet intermédiaire entre le doigt et la corde, entre le corps et l'instrument, et, par extension, entre l'esprit créateur et le matériau. Mais le titre revêt au moins un autre sens, car Rea a conçu cette œuvre comme une sorte d'hommage aux musiques extraeuropéennes, la décrivant même comme « une sorte de composition ethnologique $^{2}$ » rappelant quelque peu l'enjeu d'Exotica (1970-1971), pour instruments extra-européens, de Mauricio Kagel, où le compositeur germano-argentin a voulu, en invitant les musiciens occidentaux à jouer des instruments nonoccidentaux, "to expose the rather relative term "exoticism" "». Jouer sur la frontière entre Orient et Occident, comme Rea le fait de temps à autre ${ }^{4}$, n'est qu’une médiation de plus pour le compositeur.

Sa fascination, au cours des années 1980 et 1990, pour le postmodernisme musical (au point où un propos de John Rea est cité à la première page du «Que sais-je?» sur Musique et postmodernité ${ }^{5}$...) l'a conduit à s'inspirer d'artistes jouant avec leurs identités: par exemple, le monde étourdissant des hétéronymes de Fernando Pessoa (que Rea explore dans la musique de la pièce Les trois derniers jours de Fernando Pessoa [1997], mise en scène par Denis Marleau) ou ce célèbre tableau - un modèle d'autoréflexivité - de Velasquez, Las Meninas (exploré par Rea dans la pièce pour piano du même nom, en 1990-1991, qui, en une autre couche intertextuelle, propose des variations sur les Kinderszenen de Schumann), ou encore la géométrie paradoxale de M.C. Escher (dans Treppenmusik [1982]). Néanmoins, quiconque réduirait l'œuvre purement et simplement au postmodernisme musical se rendrait coupable d'un raccourci inadmissible! Certes, une œuvre comme Alma \& Oskar (mélodrame d'outre-tombe) (1994) se révèle un chef-d'œuvre d'allusions intertextuelles, trait que l'on associe à ce courant, comme le révèle Julien Bilodeau dans le Cahier d'analyse du présent numéro. Mais, là encore, ne retenir de
1. Voir à ce sujet John Rea (2008), "The Sorcerer as Apprentice: His Notes (The Computer in/and Teaching the Craft of Composition ", Circuit, musiques contemporaines, vol. 18, $n^{\circ}$ 1, p. 72-91.
2. Citation tirée d'une entrevue radiophonique sur $\mathrm{CBC}$, le 29 septembre 1985, en préambule d'un concert pendant lequel l'œuvre fut présentée au Vancouver East Cultural Centre et interprétée par des musiciens de la Vancouver New Music Society. L'enregistrement se trouve sur le site du Centre de musique canadienne: $<$ www.musiccentre.ca> (consulté le 19 janvier 2016).

3. Björn Heile (2009), "Weltmusik and the Globalisation of New Music", in Björn Heile (dir.), The Modernist Legacy: Essays on New Music, Aldershot, Ashgate, p. 111

4. Par exemple, et outre Médiator, dans Reception and Offering Music (1975), dans son arrangement orchestral, en 1986, de Pulau Dewata (1977) de Claude Vivier, ou encore dans Kubla Khan (Tombeau de Vivier) (1989).

5. Béatrice Ramaut-Chevassus (1998), Musique et postmodernité, "Que sais-je?", Paris, PUF, p. 1. 
6. François Nicolas (2014), Le MondeMusique. I. L'œuvre musicale et son écoute, "Musiques $\mathrm{xx}-\mathrm{xx \textrm {I }}$ e siècles", Château-Gontier, Éditions Aedam Musicae.
Rea que cette intertextualité postmoderniste, ce serait faire abstraction de la volonté structurelle du compositeur, comme en témoignent ses fresques orchestrales Homme papillon (2001) et Icare en émoi... (2012) sur lesquelles se penche Jimmie LeBlanc. Les œuvres de Rea sont par ailleurs si hétéroclites et nombreuses - il suffit de jeter un œil au catalogue détaillé de ses œuvres préparé par Solenn Hellégouarch, où l'on trouve un opéra pour enfants, un ballet composé à l'âge de 25 ans, cinq mélodrames (un genre qu'il était temps de réhabiliter!), plusieurs œuvres pour grand orchestre ou pour ensemble, parfois avec dispositif électronique, des arrangements de Mahler, Berg, etc. -, que les réduire à un seul courant, à un seul parti pris esthétique, serait les trahir.

Pour sa part, Anne Marie Messier, en collaboration avec Michel Robert, propose un portrait intellectuel de ce «musicien-pensif», comme dirait François Nicolas ${ }^{6}$, de façon hautement originale, soit en parcourant la bibliothèque du compositeur. De plus, dans l'enquête du numéro préparée par Maxime McKinley, divers acteurs de la scène canadienne de musique contemporaine partagent leurs souvenirs de leur ami, mentor, collègue et/ou collaborateur. Mais la charpente du numéro siège au creux de deux articles signés par John Rea lui-même: deux entretiens avec le musicologue renommé Ferdinand Larven Niemantz, prolongeant de la sorte un premier dialogue publié dans le vol. 9, $\mathrm{n}^{\circ} 2$ (1998) de la revue. Les illustrations de Nicholas Voeikoff-Erens complètent le dossier thématique par un jeu sur une mise en abyme des numéros de Circuit: quel meilleur hommage est-il possible de concevoir pour ce compositeur porteur de masques? Enfin, le numéro s’achève sur quelques «Nouveautés en bref» concoctées par Cléo Palacio-Quintin.

Pour terminer, je saisis également l'occasion de cette introduction pour annoncer mon départ de la direction de Circuit après dix années de service (2006-2016) - une aventure inoubliable en 28 numéros! Je souhaite tous mes vœux à mon successeur et ami, le compositeur (et membre du Comité de rédaction de la revue) Maxime McKinley, qui prendra les rênes de Circuit à partir du prochain numéro.

Bonne lecture!

Montréal, janvier 2016

Supplément web : vous pouvez retrouver l'ensemble des articles de John Rea publiés avant 2016 dans la revue Circuit, et disponibles au téléchargement, à l'adresse suivante : <www.revuecircuit.ca/collection/26_1>. 